\title{
Shear-Induced Demixing in a Polystyrene/Poly(vinyl methyl ether) Blend: In-Situ Fluorescence and Rheometry
}

\author{
Suresh Mani, Michael F. Malone," and H. Henning Winter* \\ Chemical Engineering Department, University of Massachusetts, \\ Amherst, Massachusetts 01003-0011 \\ Received February 27, 1992; Revised Manuscript Received July 10, 1992
}

\begin{abstract}
In miscible blends of polystyrene with poly(vinyl methyl ether), a shear-induced demixing occurs at temperatures as much as $40 \mathrm{~K}$ below the lower critical solution temperature. Demixing occurs at relatively low shear rates ranging from 1.5 to $4 \mathrm{~s}^{-1}$ depending on the temperature and composition; the corresponding shear stresses and first normal stress differences range from 20 to $35 \mathrm{kPa}$ and 30 to $120 \mathrm{kPa}$, respectively. There appears to be no threshold value of the shear rate or the shear stress for the onset, but a certain value of rate of work done on the sample roughly describes the steady-state demixing at a fixed temperature. When shear does induce demixing, some of the concentration differences are stable under shear for as long as $24 \mathrm{~h}$. However, in other cases, a demixing appears for a shorter time, along with relatively high stresses, shortly after the inception of shear but disappears as the stresses drop at longer times. The time scale for the development of demixing ranges from hundreds to thousands of seconds and remixing after cessation of the flow typically occurs in thousands of seconds. Particularly at temperatures near the lower critical solution temperature, a substantial time is required for the stress development, even when there is no measurable demixing as indicated by fluorescence. Transmission electron microscopy on sheared samples confirms the presence of a shear-induced structure.
\end{abstract}

\section{Introduction and Background}

Flow can strongly influence the state of mixing in polymer blends. This area is fundamentally and also technologically important, due to the large deformations and stresses that are unavoidable in processing. There have been several reports of shear-induced mixing in the polymer blends, e.g., Mazich and Carr ${ }^{11}$ and Katsaros, Malone, and Winter. ${ }^{7,8}$ Mani et al. ${ }^{10}$ reviewed additional work in this area and described fluorescence studies of shear-induced mixing in a blend of polystyrene and poly(vinyl methyl ether).

There has also been a report of flow-induced phase separation in this same polymer blend by Katsaros et al. 7,8 but little quantitative information is available. Our goals in this paper are to document the conditions for shearinduced phase separation in a miscible blend, to identify the relative importance of the stress and the shear rate, and to quantify the time scales for the growth and decay of the phase separation. Katsaros et al. ${ }^{7,8}$ studied both a planar extensional deformation in a stagnation flow die and a steady shear flow in a concentric disk rotational rheometer; optical transparency was the criterion used to identify the apparent phase transition. Extensional flow induced a phase separation in the PS/PVME blend at temperatures as much as $35 \mathrm{~K}$ below the quiescent cloud point temperature. Samples were also sheared in a parallel plate geometry at approximately $25 \mathrm{~K}$ below the quiescent cloud point temperature and rapidly quenched below the glass transition temperature; these were cloudy at the outer edge, where the shear strains were the largest, indicating a shear-induced phase separation. We know of no published reports of this phenomenon in other polymer blends.

In contrast, there have been extensive reports of shearinduced phase separation in polymer solutions near the upper critical solution temperature (UCST), e.g., Ver Strate and Phillipoff, ${ }^{16}$ Rangel-Nafaile et al., ${ }^{14}$ and Kramer-Lucas et al. ${ }^{9}$ More recently, Horst and Wolf ${ }^{6}$ described a model for the model polymer solutions exhibiting a lower critical solution temperature (LCST) and predicted a

\footnotetext{
- To whom correspondence should be addressed.
}

strongly composition-dependent shear-induced demixing, including an isolated phase-separated region.

It is important to note that quasi-thermodynamic models, which have been popular in the literature on polymer solutions, may be inadequate. This is most clearly explained by Helfand and Fredrickson, ${ }^{4}$ who gave a theory for the effects of shear on the concentration fluctuations in semidilute polymer solutions, showing that shear can dramatically enhance the concentration fluctuations even at temperatures significantly above the UCST. The effects are intimately related to the coupling of the deviatoric stresses, the concentration fluctuations, and the velocity field. Helfand and Frederickson ${ }^{4}$ also pointed out that these enhanced concentration fluctuations do not correspond to a true phase transition. (On account of this, we prefer the term "demixing" to indicate the presence of significant concentration differences in the shear field.)

Onuki ${ }^{13}$ and Milner ${ }^{12}$ have developed dynamic equations to describe the effects of shear on concentration fluctuations in more concentrated polymer solutions. These predict a negative diffusion coefficient when there is a negative slope of the first normal stress difference versus composition at constant shear stress, corresponding to a shear-induced spinodal decomposition. The shift in the apparent coexistence temperature was predicted to increase strongly with increasing first normal stress difference and with the polymer concentration. Onuki ${ }^{13}$ also suggested that shear-induced phase separation would be most pronounced in polymer blends when the chain lengths of the two polymers are considerably different.

In related experiments, $W u, P i n e$, and $D i x{ }^{19}$ sheared a semidilute solution of PS in DOP in a transparent cylindrical Couette cell and measured the light scattering. For "weak shear" $\left(\dot{\gamma}<4 \mathrm{~s}^{-1}\right)$ the shear stress was larger than the first normal stress difference and there was no enhancement in the scattering perpendicular to the shear plane, indicating that shear flow did not induce demixing. However, for "strong shear" $\left(\dot{\gamma}>4^{-1}\right)$ where the first normal stress difference was greater than the shear stress, there was a significant increase in the scattering perpendicular to the plane of shear. The measured behavior of 
the nonequilibrium structure factor was in reasonable accord with the theory of Helfand and Fredrickson. ${ }^{4}$

In summary, there are several reports of shear-induced demixing in polymer solutions where the elevation in the apparent UCST is as large as several tens of degrees. The effect is more pronounced for higher concentrations and molecular weights of the polymer and is generally thought to be related to the elasticity of the fluid. In several studies, shear-induced demixing has been described by using a quasi-equilibrium theory, but this phenomenological approach may be inadequate, principally because it does not describe the anisotropic effects of shear on the structure, as predicted by Helfand and Fredrickson ${ }^{4}$ and measured by Wu et al. 19

There are relatively little data on polymer blends, but the analysis of Onuki ${ }^{13}$ suggests that the effects should be larger in blends than in solutions, and this is consistent with the report of Katsaros et al. ${ }^{8}$ Helfand and Fredrickson ${ }^{4}$ also point out the importance of a concentrationdependent viscosity and normal stresses, which are prominent in polymer blends. There is a need for quantitative information concerning the effects of shear on polymer blends and on the magnitudes of the corresponding stresses and shear rates.

In this paper, we describe rheological and simultaneous in-situ measurements of the state of segregation and the dynamics of the approach to a steady state. We focus on the effects of a homogeneous shear field to avoid large isotropic pressures (Hiramatsu et al. ${ }^{5}$ show that the shift of the cloud point temperature due to pressure can be as large as $0.3 \mathrm{~K} / \mathrm{MPa}$, at the critical composition of a PS/ PVME blend). PS/PVME blends with a range of compositions were sheared at various constant rates and the evolution of the shear stress and first normal stress difference was measured. Simultaneously, the time scales for demixing and also for remixing after stopping the flow were obtained from in-situ fluorescence measurements, which have a sensitivity to concentration fluctuations at length scales comparable to those of small-angle neutron scattering and which have previously been used in extensive studies of the equilibrium phase behavior in PS/ PVME blends. ${ }^{1-3}$ Transmission electron microscopy was also used to study samples prepared by rapidly quenching sheared blends.

\section{Materials and Experimental Techniques}

The materials, blend preparation, apparatus, and most of the fluorescence and rheometry techniques are identical with those described by Mani et al. ${ }^{10}$ and only a brief summary is given below.

Materials. Polystyrene (Monsanto Lustrex 101) samples were used with $M_{\mathrm{W}}=259000$ and $M_{\mathrm{w}} / M_{\mathrm{N}}=1.55$. Poly (vinyl methyl ether) was obtained from Scientific Polymer Products with $M_{\mathrm{w}}$ $=85000$ and $M_{\mathrm{W}} / M_{\mathrm{N}}=1.39:$ Anthacene-labeled polystyrene (PS*), with a molecular weight of 200000 , was prepared according to the description given by Valeur et al. ${ }^{15}$ and supplied to us by J. L. Halary and L. Monnerie.

Blends of PS and PVME with 1 wt \% of PS* were prepared by solvent casting from toluene. The use of fluorescence measurements to determine the phase transition temperatures under quiescent conditions and to study mixing during shear flow was described by Mani et al. ${ }^{10}$ The resulting equilibrium phase diagram is reproduced in Figure 1, for comparison with subsequent results. The glass transition temperatures are practically independent of molecular weight for high molecular weight PS/PVME blends and their values were adopted from the measurements of Yang et al. ${ }^{20}$

Rheometry. A Rheometrics RMA- 800 rheometer was modified for improved temperature control, to include guarding of the sample in a zero temperature gradient region, and a fiber optic probe was flush-mounted on the plate in a cone-and-plate

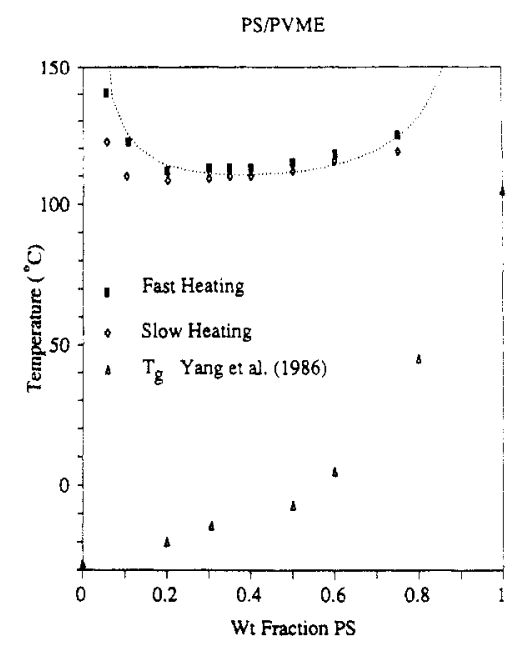

Figure 1. Equilibrium phase diagram for PS/PVME blends as reported by Mani et al. ${ }^{10}$ The glass transition temperatures are from Yang et al. ${ }^{20}$

configuration for in-situ fluorescence measurements. The entire sample was protected by a dry nitrogen environment. See Mani et al. ${ }^{10}$ for further discussion and a schematic of the equipment. Most experiments were done with plates of radius $6 \mathrm{~mm}$, for which the torque and normal force limitations of the rheometer do not allow measurements at shear stresses greater than approximately $40 \mathrm{kPa}$ or for first normal stress differences greater than approximately $125 \mathrm{kPa}$. We were also limited to shear rates of approximately $8 \mathrm{~s}^{-1}$ in order to maintain stable shear flows (for a discussion of the instability at higher shear rates see Walters ${ }^{18}$ ).

Electron Microscopy. To prepare solid-state samples of sheared blends, disposable plates were used in the rheometer and the samples were removed and quenched in liquid nitrogen; this took approximately $15 \mathrm{~s}$ after cessation of the flow. The plates were separated carefully and triangular wedges were cut at a location approximately two-thirds of the plate radius from the center of the sample to avoid edge effects. TEM sections with a thickness of approximately $0.1 \mu \mathrm{m}$ were obtained, perpendicular to the flow direction, by cryo-microtoming these wedges at $-100^{\circ} \mathrm{C}$.

The contrast between the phases in the PS/PVME system does not occur by diffraction or scattering because both phases are amorphous and the differences in atomic weight per unit volume are small. However, the polymers happen to degrade selectively in the electron beam (e.g., Voigt-Martin et al. ${ }^{17}$ ); the light regions in the micrographs are the PVME-rich regions and the dark are the PS-rich regions. The images were obtained with a JEOL-100CX electron microscope, using a high accelerating voltage of $100 \mathrm{kV}$, for which the contrast between the phases developed in approximately 15 to $20 \mathrm{~s}$ and then did not change significantly over $30 \mathrm{~min}$.

\section{Results}

Samples with compositions ranging from $10 / 90$ to $60 / 40$ (w/w) PS/PVME were equilibrated in the rheometer for $2-3 \mathrm{~h}$ at the desired temperature for subsequent experiments until the fluorescence intensity reached a steadystate value. The samples were then sheared at a constant rate after an impulsive start of the lower cone, and the torque, normal force, and fluorescence intensity $\left(I_{f}\right)$ were measured; each run was repeated at least twice on different samples. The shear stress, $\tau$, and the first normal stress difference, $N_{1}$, were calculated from the measured torque and normal force and the flow geometry by standard methods.

In general, the stress levels could be raised by increasing the shear rate (up to the stability limit), by lowering the temperature, and/or by choosing samples with a high PS content.

Steady-State Stresses and Fluorescence. Table I shows that shear rates in the range 1-8 $\mathrm{s}^{-1}$ do not result 
Table I

Fluorescence and Stress Results at Steady State for a 20/80 Blend

\begin{tabular}{cccccc}
\hline$T,{ }^{\circ} \mathrm{C}$ & $\begin{array}{c}\text { fluorescence } \\
\text { intensity }\end{array}$ & $\dot{\gamma}, \mathrm{s}^{-1}$ & $\tau, \mathrm{kPa}$ & $N_{\mathrm{l}}, \mathrm{kPa}$ & $\tau \dot{\gamma}, \mathrm{kPa} / \mathrm{s}$ \\
\hline 105 & 1.0 & 1.0 & 2 & 2 & 2 \\
& 1.0 & 2.0 & 3 & 3 & 6 \\
& 1.0 & 4.0 & 5 & 6 & 20 \\
\multirow{3}{*}{80} & 1.0 & 8.0 & 12 & 18 & 96 \\
& 1.0 & 1.0 & 6 & 15 & 6 \\
& 1.0 & 2.0 & 16 & 30 & 32 \\
& 1.5 & 4.0 & 25 & 45 & 100 \\
45 & 1.8 & 6.0 & 32 & 57 & 192 \\
& 1.0 & 0.1 & 18 & 36 & 1.8 \\
& 1.0 & 0.2 & 30 & 75 & 6
\end{tabular}

Table II

Fluorescence and Stress Results at Steady State for a 40/60 PS/PVME Blend

\begin{tabular}{cccccc}
\hline$T,{ }^{\circ} \mathrm{C}$ & $\begin{array}{c}\text { fluorescence } \\
\text { intensity }\end{array}$ & $\dot{\gamma}, \mathbf{s}^{-1}$ & $\tau, \mathbf{k P a}$ & $N_{1}, \mathbf{k P a}$ & $\tau \dot{\gamma}, \mathbf{k P a} / \mathbf{s}$ \\
\hline 105 & 1.0 & 1.0 & 9 & 20 & 9 \\
& 1.0 & 2.0 & 13 & 28 & 26 \\
\multirow{3}{*}{95} & 2.0 & 4.0 & 26 & 70 & 104 \\
& 1.0 & 0.5 & 6 & 17 & 3 \\
& 1.0 & 1.0 & 9 & 23 & 9 \\
& 1.5 & 2.0 & 20 & 60 & 40 \\
& 1.8 & 3.0 & 22 & 68 & 66 \\
80 & 2.3 & 4.0 & 30 & 100 & 120 \\
& 2.3 & 5.0 & 32 & 118 & 160 \\
& 1.0 & 0.5 & 9 & 21 & 4.5 \\
& 1.0 & 1.0 & 16 & 41 & 16 \\
& 1.4 & 1.5 & 20 & 57 & 30 \\
& 1.7 & 2.0 & 25 & 102 & 50 \\
& 2.0 & 2.5 & 31 & 122 & 77.5
\end{tabular}

in an increased fluorescence in the $20 / 80$ blend at a temperature of $105^{\circ} \mathrm{C}$, slightly below the LCST. At 80 ${ }^{\circ} \mathrm{C}$ and low shear rates, the behavior is similar, even though the first normal stress difference is substantially larger than the shear stress. However, at larger shear rates of 4 and $6 \mathrm{~s}^{-1}$, there is evidence of significant demixing in the increased fluorescence intensities. At $45^{\circ} \mathrm{C}$, relatively large values of $\tau$ and $N_{1}$ ( 30 and $75 \mathrm{kPa}$, respectively) did not result in any measurable demixing. (The shear rates at $45^{\circ} \mathrm{C}$ were limited to less than $0.3 \mathrm{~s}^{-1}$ on account of the maximum torque and normal force limitations in the rheometer.)

For the $40 / 60$ blend (Table II), shear rates up to $2 \mathrm{~s}^{-1}$ at $105^{\circ} \mathrm{C}$ did not change the fluorescence intensity, similar to the results for the $20 / 80$ blend at the same temperature (Table I). However, at a shear rate of $4 \mathrm{~s}^{-1}, \tau$ and $N_{1}$ were 26 and $70 \mathrm{kPa}$ and the steady-state fluorescence intensity was doubled. At lower temperatures, the demixing appears at lower shear rates of 1.5 to $2.0 \mathrm{~s}^{-1}$, corresponding to shear stresses of $20-31 \mathrm{kPa}$ and first normal stress differences of $57-122 \mathrm{kPa}$.

Table III, along with some entries from Tables I and II, gives steady-state results at $80^{\circ} \mathrm{C}$ for a wide range compositions and shear rates. A shear rate of $1.5 \mathrm{~s}^{-1}$ results in no measurable demixing in the $60 / 40$ blend, in contrast to the results for the $50 / 50$ and $40 / 60$ blends at the same temperature; in fact, $\tau$ and $N_{1}$ were identical for these cases, within the experimental error.

Dynamics. Information on the evolution of the stresses and fluorescence is important to determine the time scales of demixing and remixing and to confirm the existence of a steady state. Figure 2 shows the evolution of the stresses and the fluorescence in a $20 / 80$ blend at $105{ }^{\circ} \mathrm{C}$, close to the equilibrium phase transition temperature of $110^{\circ} \mathrm{C}$. Shear rates of 1 to $8 \mathrm{~s}^{-1}$ caused no measurable change in the fluorescence, but the stresses show a relatively long
Table III

Steady-State Fluorescence and Stress Results at $80^{\circ} \mathrm{C}$

\begin{tabular}{cccccc}
\hline $\begin{array}{c}\text { PS/PVME, } \\
\text { w/w }\end{array}$ & $\begin{array}{c}\text { fluorescence } \\
\text { intensity }\end{array}$ & $\dot{\gamma}, \mathrm{s}^{-1}$ & $\tau, \mathbf{k P a}$ & $N_{\mathbf{l}}, \mathbf{k P a}$ & $\tau \dot{\gamma}, \mathbf{k P a} / \mathbf{s}$ \\
\hline $10 / 90$ & 1.0 & 2.0 & 10 & 15.4 & 20 \\
& 1.4 & 5.0 & 20 & 30.5 & 100 \\
$30 / 70$ & 1.0 & 2.0 & 10 & 20.0 & 20 \\
& 1.4 & 4.0 & 20 & 45 & 80 \\
& 1.6 & 5.5 & 30 & 75 & 165 \\
$50 / 50$ & 1.0 & 1.0 & 10 & 21 & 10 \\
& 1.2 & 1.5 & 20 & 56.4 & 30 \\
$60 / 40$ & 1.0 & 1.5 & 20 & 57 & 30 \\
& 1.2 & 2.5 & 30 & 92 & 75 \\
& 1.8 & 3.0 & 36 & 110 & 108
\end{tabular}

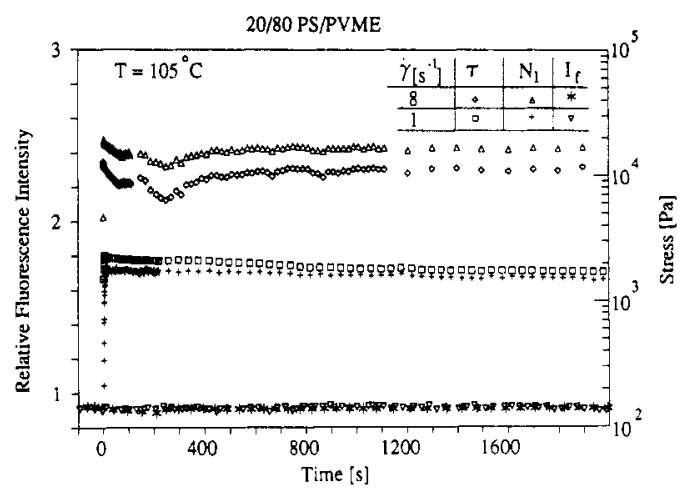

Figure 2. Shear stress $(\tau)$, first normal stress difference $\left(N_{1}\right)$, and fluorescence intensity $\left(I_{f}\right)$ for a $20 / 80$ blend, sheared at 1 and $8 \mathrm{~s}^{-1}$ and $105^{\circ} \mathrm{C}$.

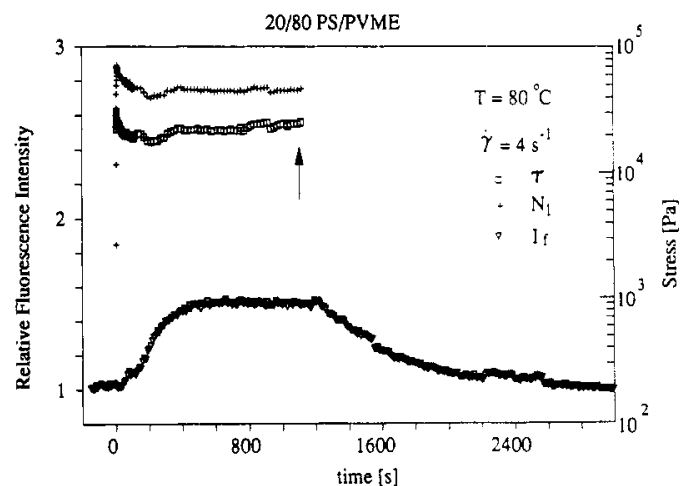

Figure 3. Shear stress ( $\tau$ ), first normal stress difference $\left(N_{1}\right)$, and fluorescence intensity $\left(I_{f}\right)$ for a $20 / 80$ blend, sheared at $4 \mathrm{~s}^{-1}$, at $80^{\circ} \mathrm{C}$.

transient before reaching a steady state in approximately $1000 \mathrm{~s}$. The overshoot is small and the transient is smooth at a shear rate of $1 \mathrm{~s}^{-1}$; at a higher shear rate of $8 \mathrm{~s}^{-1}$, the overshoot is larger and there are significant oscillations in $\tau$ and $N_{1}$.

At a lower temperature of $80^{\circ} \mathrm{C}$ and shear rates of 1 and $2 \mathrm{~s}^{-1}$, the stress response was qualitatively similar and there was no measurable change in the fluorescence intensity. However, at $4 \mathrm{~g}^{-1}$ (Figure 3), the fluorescence intensity increased monotonically by $50 \%$ in approximately $500 \mathrm{~s}$ and there was an overshoot in the stresses at short times, followed by oscillations for several hundred seconds. After cessation of the flow, the stresses dropped rapidly but the fluorescence intensity returned to its original level only after approximately $1500 \mathrm{~s}$. At a higher shear rate of $6 \mathrm{~s}^{-1}$, the behavior was similar and the fluorescence intensity increased by $80 \%$ in to a steady state in approximately $300 \mathrm{~s}$. At $45^{\circ} \mathrm{C}$, the maximum accessible shear rate was approximately $0.2 \mathrm{~s}^{-1}$; the evolution of $\tau$ and $N_{1}$ was smoother than at temperatures closer to the coexistence temperature and the stress reached steady state in approximately $50 \mathrm{~s}$ as shown in Figure 4. We could not 


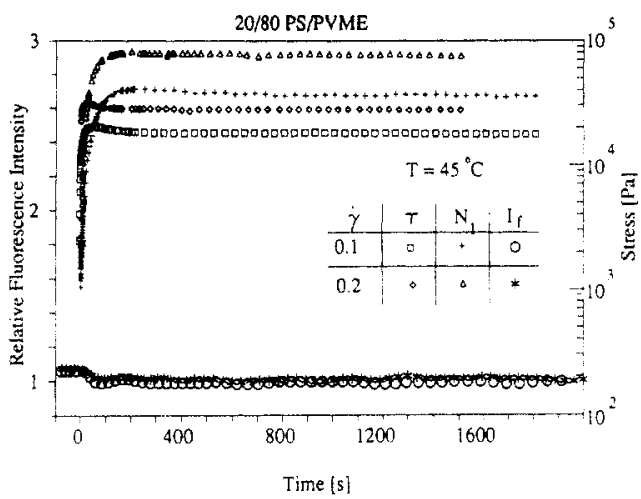

Figure 4. Shear stress ( $\tau$ ) first normal stress difference $\left(N_{1}\right)$, and fluorescence intensity $\left(I_{f}\right)$ for a $20 / 80$ blend, sheared at 0.1 and $0.2 \mathrm{~s}^{-1}$ and $45^{\circ} \mathrm{C}$.

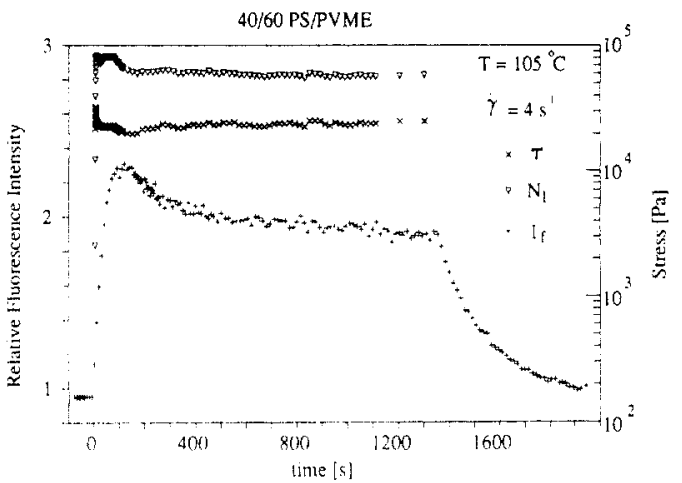

Figure 5. Shear stress $(\tau)$, first normal stress difference $\left(N_{1}\right)$, and fluorescence intensity $\left(I_{f}\right)$ for a $40 / 60$ blend, sheared at $4 \mathrm{~s}^{-1}$ and $105^{\circ} \mathrm{C}$.

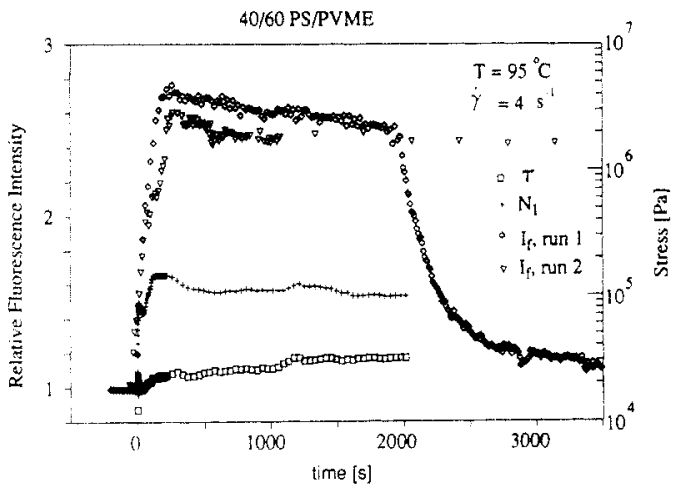

Figure 6. Shear stress $(\tau)$, first normal stress difference $\left(N_{1}\right)$ and fluorescence intensity $\left(I_{f}\right)$ for a $40 / 60$ blend, sheared at $4 \mathrm{~s}^{-1}$ and $95^{\circ} \mathrm{C}$. The run was repeated for $24 \mathrm{~h}$ (Figure 7); the fluorescence data for the first hour is included in this figure for comparison.

examine higher stresses in any of these cases, on account of the torque and normal force limitations in the rheometer.

For the $40 / 60$ blend, at $105^{\circ} \mathrm{C}$ and low shear rates, the evolution of $\tau$ and $N_{1}$ was similar to that described for the $20 / 80$ blend at $105^{\circ} \mathrm{C}$, and the fluorescence intensity showed no evidence of demixing. However, at larger shear rates such as those shown in Figure 5, the fluorescence intensity increased substantially with an initial overshoot of more than $100 \%$ and a very slow approach to a steady state after $1000 \mathrm{~s}$ or more. At a lower temperatures, the behavior was similar but the increase in the fluorescence was larger and the stresses showed a longer transient, e.g., Figure 6 (note the change in scale for the stresses). Upon cessation of shear, the fluorescence intensity returned to its original level in approximately $1000 \mathrm{~s}$. This experiment was repeated for $24 \mathrm{~h}$ on a different sample with the same composition, but without stopping the shear, giving the

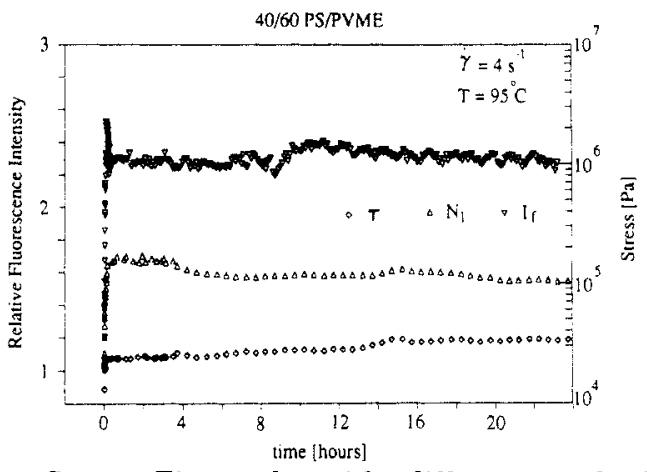

Figure 7. Same as Figure 7, but with a different sample, sheared for $24 \mathrm{~h}$.

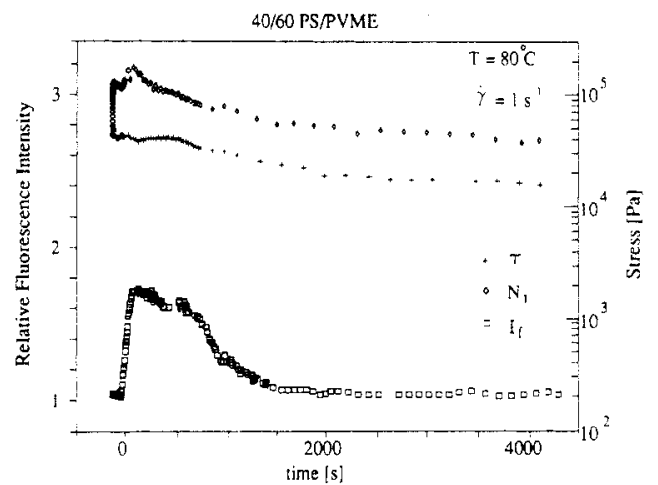

Figure 8. Shear stress ( $\tau$ ), first normal stress difference $\left(N_{1}\right)$ and fluorescence intensity $\left(I_{f}\right)$ for a $40 / 60$ blend, sheared at $1 \mathrm{~s}^{-1}$ and $80^{\circ} \mathrm{C}$.

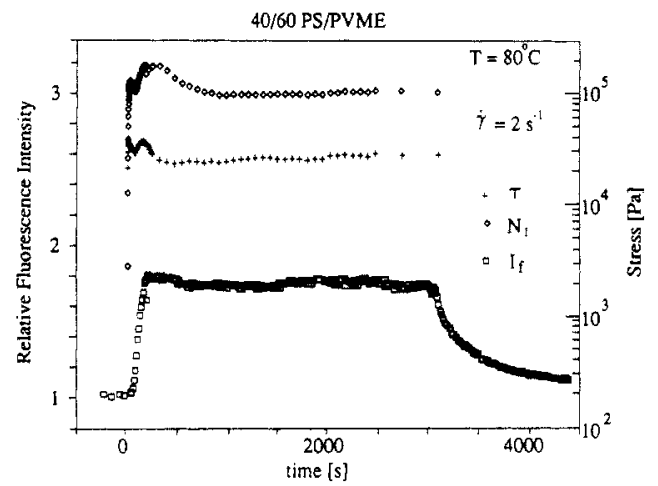

Figure 9. Shear stress ( $\tau)$, first normal stress difference $\left(N_{\mathrm{l}}\right)$, and fluorescence intensity $\left(I_{f}\right)$ for a $40 / 60$ blend, sheared at $2 \mathrm{~s}^{-1}$ and $80^{\circ} \mathrm{C}$.

results shown in Figure 7. The fluorescence intensity remained at approximately $230 \%$ of its original level and the stresses showed a very long transient. The time scale for remixing on stopping the flow was independent of whether the blend was sheared for 1 or $24 \mathrm{~h}$.

The most interesting transient behavior was observed for the $40 / 60$ blend as shown in Figure 8 . At $80^{\circ} \mathrm{C}$ and a shear rate of $1 \mathrm{~s}^{-1}$, the fluorescence intensity increased by $70 \%$ in approximately $200 \mathrm{~s}$, accompanied by the second of two maxima in the stresses. After what appear to be small oscillations, the stresses and fluorescence both decrease over a period of approximately $1500 \mathrm{~s}$ with the fluorescence returning to its original level. At a somewhat higher shear rate of $2 \mathrm{~s}^{-1}$ (Figure 9 ), the steady state shows an elevated fluorescence and significant demixing. The stresses again had two overshoots, with the second maximum accompanying the approach of fluorescence to steady state in approximately $500 \mathrm{~s}$. On stopping the flow, the fluorescence intensity recovered its original level in approximately $1000 \mathrm{~s}$. 


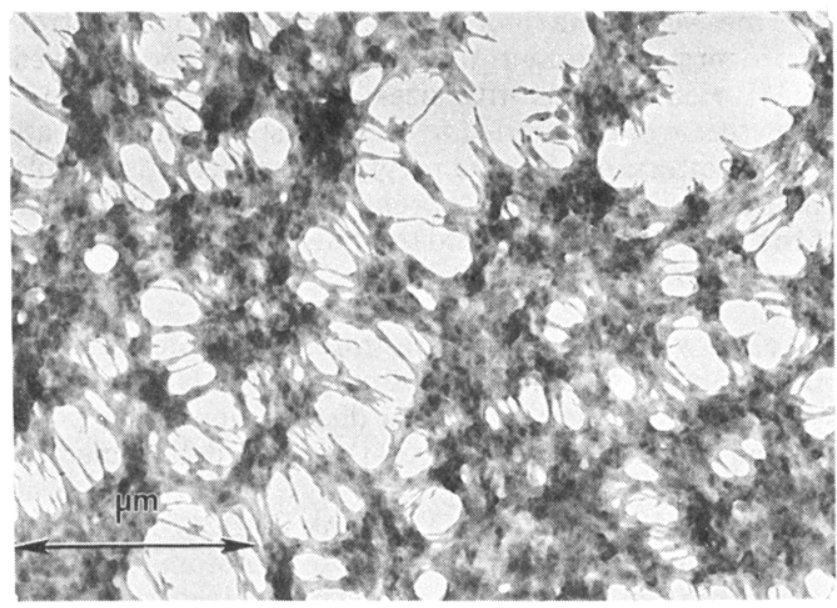

Figure 10. TEM for sections normal to the flow direction for a $20 / 80$ blend sheared at a $4 \mathrm{~s}^{-1}$ and $80^{\circ} \mathrm{C}$, then quenched immediately after cessation of the flow.

Electron Microscopy. A 20/80 sample was maintained in the miscible state at $80^{\circ} \mathrm{C}$ for $2 \mathrm{~h}$, without shear; the equilibrated sample was quenched and electron micrographs were taken of the cryo-microtomed sections. No structure was detected in any of the sections, indicating that the quenching process did not produce any unexpected effects in the blend.

For the sample and conditions corresponding to the steady state in Figure 4, a typical TEM image of a crosssection perpendicular to the flow direction is shown in Figure 10. The structure is quite complex, with interconnected PS-rich regions and disconnected lighter PVMErich regions with sizes in the range 0.1-1 $\mu \mathrm{m}$. On this scale, there seems to be little or no anisotropy and this was typical of the other micrographs taken in this cross-section.

\section{Discussion}

In-situ measurements show a large increase in the fluorescence intensity under shear, which is interpreted as shear-induced demixing. The fluorescence persists for as long as $24 \mathrm{~h}$, e.g., Figure 7 (approximately $100 \mathrm{~K}$ above the $T_{\mathrm{g}}$ ), showing that a stable, segregated phase structure can be formed.

TEM on rapidly quenched samples shows a considerable interconnectivity in the PS-rich phase, suggesting that the mechanism of phase separation could be spinodal decomposition as proposed by Onuki, ${ }^{13}$ but dynamic information on the structure would be required to confirm this mechanism.

There are two distinct regimes for the effects of shear on the apparent coexistence temperature of the blend as shown in Figure 11, which gives the temperature elevation as function of the shear stress. (Positive values of the temperature difference correspond to shear-induced mixing and were taken from the measurements of Mani et al. $\left.{ }^{10}\right)$. At low values of the shear stress, the apparent coexistence temperature shifts to higher temperatures; Mani et al. ${ }^{10}$ correlated this shear-induced mixing with the shear rate according to $\Delta T / T_{\mathrm{s}}=(0.015 \pm$ $0.002) \dot{\gamma}^{0.59 \pm 0.04}$ independent of the composition in the range $20-60 \%$ PS. Negative values of the temperature elevation in Figure 11 correspond to shear-induced demixing, which is induced at values of the shear stress greater than approximately $20 \mathrm{kPa}$. Significantly larger values of the shear stress are required to induce phase separation at lower temperatures (but these stresses are not unusually large compared to those found in many practical situations). This suggests a dynamic balance

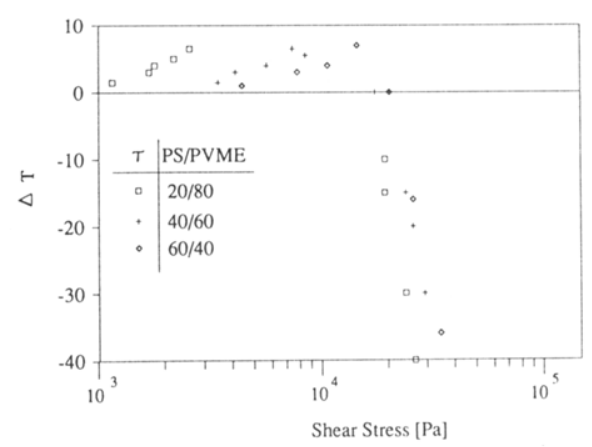

Figure 11. Shift in the apparent phase transition temperatures vs shear stress; the data on shear-induced mixing were taken from Mani et al. ${ }^{10}$

between a tendency for phase separation arising from high stresses and a tendency for mixing, arising from high shear rates. The transient nature of the demixing under certain circumstances, e.g., Figure 8, and especially its relationship to the stress levels also support this view.

Tables I-III show that the shear rates required to induce phase separation depended significantly on the composition; e.g., at $80^{\circ} \mathrm{C}$ a $20 / 80$ blend required a shear rate of $4 \mathrm{~s}^{-1}$ but the $40 / 60$ blend required a shear rate of only $1.5 \mathrm{~s}^{-1}$. Table I also shows that a shear stress of $30 \mathrm{kPa}$ and first normal stress difference of $75 \mathrm{kPa}$ induced phase separation at $80^{\circ} \mathrm{C}$ in the $20 / 80$ blend but did not induce phase separation at $45^{\circ} \mathrm{C}$, showing that there is no critical value of either the shear stress or the first normal stress difference for demixing. At $80^{\circ} \mathrm{C}$ and identical shear rates of $1.5 \mathrm{~s}^{-1}$, the steady-state stress response of the 40/60 and the $60 / 40$ blends is essentially identical, but only the 40 / 60 blend was demixed, with a fluorescence increase of $40 \%$. This suggests that the demixing may be very sensitive in this region, if not necessarily abrupt.

The demixing depends strongly on both the shear rate and the shear stress, but large stresses or shear rates alone are not sufficient to cause demixing. It is useful to examine the product of the shear stress and shear rate, i.e., the rate of work done the sample per unit volume. Tables I-III tabulate this quantity at steady state and we note that demixing was induced for all blends (10-60\% PS) at 80 ${ }^{\circ} \mathrm{C}$, for $\tau \dot{\gamma}>30 \mathrm{kPa} / \mathrm{s}\left[0.072 \mathrm{cal} /\left(\mathrm{cm}^{3} \mathrm{~s}\right)\right]$. At higher temperatures, the value of $\tau \dot{\gamma}$ associated with demixing seems to be somewhat larger. We also note that the value of $\tau \dot{\gamma}$ calculated from steady-state data can be applied to interpret some of the transient effects as can be seen by examination of Figures 8 and 9 . While this relationship is approximate and motivated by quasi-thermodynamic ideas, a more detailed understanding will require a treatment for blends similar to the theory of Helfand and Fredrickson ${ }^{4}$ for solutions. We also note that the secondorder fluid description of the rheology may be inadequate in such a theory for blends.

Figure 2 shows that the stress requires approximately $1000 \mathrm{~s}$ to reach a steady state at $105^{\circ} \mathrm{C}$, which may be due to the proximity to the critical conditions and the associated concentration fluctuations. At temperatures much lower than the LCST, the evolution of the stress was faster and smoother, as shown in Figure 4. However, the fluorescence intensity remained at its original level in both cases, suggesting that the fluctuations were not enhanced by shear, at least not on a scale that would cause a significant increase in fluorescence. Of course, if the amplitudes were unchanged, the concentration field could be made anisotropic by shear without necessarily changing the fluorescence as measured here. Anisotropic concentration fluctuations could alter the mechanical response 


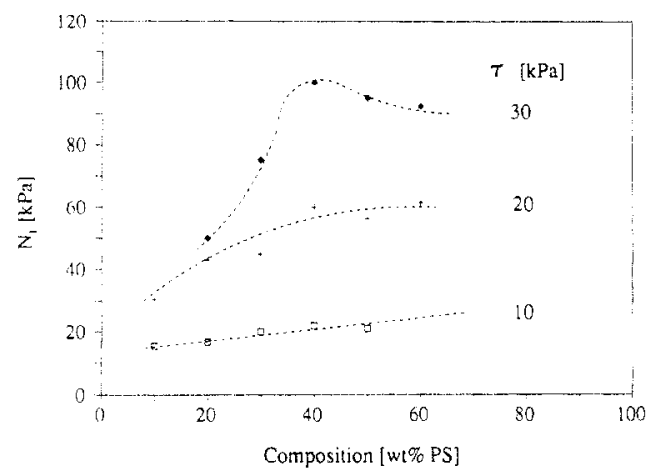

Figure 12. Steady-state composition dependence of the first normal stress difference $\left(N_{1}\right)$ at $80^{\circ} \mathrm{C}$ and constant shear stress $(\tau)$.

of the blend on account of concentration-dependent viscosity and normal stresses.

Figure 12 shows $N_{1}$ versus the weight fraction of PS at approximately constant $\tau$, prepared by cross-plotting the steady-state data from Tables I-III at $80^{\circ} \mathrm{C}$. Shearinduced demixing was observed at and near the maximum in the apparent $N_{1}$ for $\tau=30 \mathrm{kPa}$; similar behavior has been reported in polymer solutions, e.g., Rangel-Nafaile et al. ${ }^{14}$ (Figure 6). The negative slope in the same region is also consistent with the previous experiments of Katsaros et al. ${ }^{8}$ on the same blend and in agreement with the predictions of Onuki. ${ }^{13}$

\section{Conclusions}

In miscible PS/PVME blends, a shear-induced demixing occurs at temperatures as much as $40 \mathrm{~K}$ below the LCST. The shear rates required to induce demixing range from 1.5 to $4 \mathrm{~g}^{-1}$ and depend on the composition and temperature; the corresponding shear stress ranges from 20 to 35 $\mathrm{kPa}$ and the first normal stress difference ranges from 30 to $120 \mathrm{kPa}$. There appears to be no threshold value of the shear rate or the shear stress for the onset of demixing, but a certain value of rate of work done on the sample roughly describes the data at a fixed temperature. When shear does induce demixing, the fluroescence intensity in some cases remains much higher than that of the quiescent blend for as long as $24 \mathrm{~h}$, showing that the segregated phase structures can be stable under shear. However, in other cases, demixing appears only for a time along with relatively high stresses shortly after the inception of shear but disappears as the stresses drop at longer times. The time scale for the development of demixing ranges from hundreds to thousands of seconds, depending on the composition and temperature; after cessation of the flow, remixing typically occurs in thousands of seconds. Especially at temperatures near the LCST, a substantial time is required for the stress development, even when there is no increase in the fluorescence. Transmission electron microscopy confirmed phase separation and showed PVME-rich domains with sizes in the range 0.1-1 $\mu \mathrm{m}$.

Further work is needed to determine how large shearinduced effects are in other polymer blends, both miscible and immiscible. A more thorough study of the influence of the molecular weights and their distribution would also be valuable.

Acknowledgment. We are grateful to Professors J. L. Halary and L. Monnerie for supplying the labeled polystyrene. The molecular weight distribution of the polystyrene and poly(vinyl methyl ether) was determined by gel permeation chromatography by Dr. J. Swadesh at Polymer Labs, Amherst. We thank D. B. Scott for her help in sample preparation for cryo-microtoming and in taking the first few micrographs. This project was funded by Center for University of Massachusetts Industrial Research on Polymers and by the donors of the Petroleum Research Fund, administered by the American Chemical Society.

\section{References and Notes}

(1) Halary, J. L.; Ubrich, J. M.; Monnerie, L.; Yang, H.; Stein, R. S. Poly. Commun. 1985, 26, 73.

(2) Halary, J. L.; Ubrich, J. M.; Nunzi, J. M.; Monnerie, L.; Stein, R. S. Polymer 1984, 25, 956.

(3) Halary, J. L.; Monnerie, L. In Photophysical and Photochemical Tools in Polymer Science; Winnik, M. A., Ed.; D. Reidel: Dordrecht: The Netherlands, 1986; $p 589$.

(4) Helfand, E.; Frederickson, G. H. Phys. Rev. Lett. 1989, 62, 2468.

(5) Hiramatsu, N.; Hashida, S.; Yasuniwa, M.; Hirakawa, S. Fukuoka Univ. Sci. Rep. 1983, 13, 39.

(6) Horst, R.; Wolf, B. A. Macromolecules 1991, 24, 2236.

(7) Katsaros, J.D.; Malone, M. F.; Winter, H. H. Polym. Bull. 1986, $16,83$.

(8) Katsaros, J. D.; Malone, M. F.; Winter, H. H. Polym. Eng. Sci. $1989,29,1434$.

(9) Kramer-Lucas, H.; Schenck, H.; Wolf, B. A. Makromol. Chem. $1988,189,1613$.

(10) Mani, S.; Malone, M. F.; Winter, H. H.; Halary, J. L.; Monnerie, L. Macromolecules 1991, 24, 5451.

(11) Marich, K. A.; Carr, S. H.; J. Appl. Phys. 1983, 54, 5511.

(12) Milner, S. T. Phys. Rev. Lett. 1991, 66, 1477.

(13) Onuki, A. J. Phys. Soc. Jpn. 1990, 59, 3427.

(14) Rangel-Nafaile, C.; Metzner, A. B.; Wissbrun, K. F. Macromolecules 1984, 17, 1187.

(15) Valeur, B.; Monnerie, L.; Rempp, J. C. R. Acad. Sci. Paris 1974, C270, 1009 .

(16) Ver Strate, G.; Phillipoff, W. J. Polym. Sci., Polym. Lett. 1974, $12,267$.

(17) Voigt-Martin, I. G.; Leister, K. H.; Roseau, R.; Koningsveld, R. J. Polym. Sci., Polym. Phys. 1986, 24, 723.

(18) Walters, K. In Rheometry; Wiley, New York, 1975; p 85.

(19) Wu, X. L.; Pine, D. J.; Dixon, P. K Phys. Rev. Lett. 1991, 66, 2408.

(20) Yang, H.; Shibayama, H.; Stein, R. S.; Shimuzu, N.; Hashimoto, T. Macromolecules 1986, 19, 1667.

Registry No. PS (homopolymer), 9003-53-6; PVME (homopolymer), 9003-09-2. 